\title{
DIGITALE WERKINTEGRITÄT: DER EUROPÄISCHEN WERKBEGRIFF UND DER MITGLIEDSTAATLICHE SCHUTZ VOR WERKVERNICHTUNG
}

\author{
Clemens Thiele
}

\begin{abstract}
Das Urheberrecht war und ist dem Jubilar stets ein Anliegen. Es schlägt auch die Brücke zum Verfasser dieses Beitrags. Als junger «Praktiker» hatte ich 2001 erstmals Gelegenheit am "4. Internationalen Rechtsinformatik Symposium» 1 teilzunehmen, zum urheberrechtlichen Thema «Content-Krieg im Web» vorzutragen und zu publizieren. Schon damals war Erich Schweighofer nicht nur als Organisation-Lead «Auf dem Weg zur ePerson»² tätig, sondern hat selbst unermüdlich die Veranstaltung fotografierend dokumentiert - (digitales) Werkschaffen at its best. Was liegt da näher, als dem Geehrten einen Beitrag zu widmen, der sich mit den Grundfragen und dem Grundverhältnis des Werkes an sich sowie der Werkerhaltung befasst. Ad multos annos!
\end{abstract}

\section{Inhaltsverzeichnis}

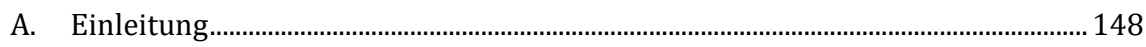

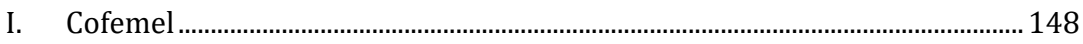

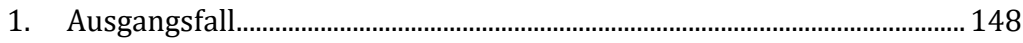

2. Entscheidung des Gerichts ..................................................................... 150

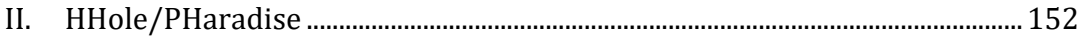

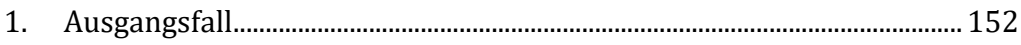

2. Entscheidung des Gerichts ....................................................................... 153

1 Den damaligen «Salzburger Rechtsinformatik-Gesprächen», die nunmehr längst zur unverzichtbaren Instititution «Internationales Rechtsinformatik Symposion (IRIS)» geworden sind.

2 So der Titel des von Schweighofer/MEnzel/KreuZBAuer herausgegegebenen Tagungsbandes aus dem Jahr 2001. 
B. Europäischer Werkbegriff...........................................................................................154

I. Urheberrechtliches Werk iS des Unionsrechts..................................................155

1. Merkmal der objektiven Identifizierung ......................................................155

2. Kriterium der Originalität..........................................................................156

II. Abgrenzungen zu Nicht-Werken .....................................................................158

C. Mitgliedstaatlicher Werkschutz .................................................................................159

I. Unionsrechtliche Grundlagen .............................................................................160

II. Verletzung von $\S 21$ UrhG - Kein Zerstörungsschutz in Österreich?............161

1. Grundlagen und Regelungsgehalt................................................................161

2. Begriff des Werkschutzes.................................................................................163

3. Schutz der Werkintegrität auch ohne Öffentlichkeit .................................163

4. Zulässige Einschränkungen...........................................................................164

5. Absolutes Änderungsverbot - Entstellungsschutz .....................................167

6. Schutz vor der Zerstörung des Werkes .........................................................168

D. Zusammenfassung .........................................................................................................169

\section{A. EINLEITUNG}

Jüngst entschiedene Fälle des EuGH und des BGH bieten den aktuellen Anlass, sich mit dem unionsrechtlichen Werkbegriff und dem urheberpersönlichkeitsrechtlichen Schutz vor Werkzerstörung nach dem Recht der Mitgliedstaaten auseinanderzusetzen.

\section{Cofemel ${ }^{3}$}

\section{Ausgangsfall}

Der Ausgangsfall spielt in Portugal. Übrigens ein Land, das der Jubilar auch immer wieder gern bereist und ob seiner Kultur und Gastlichkeit sehr schätzt.

3 EuGH 12.9.2019, C-683/17 (Cofemel - Sociedade de Vestuário SA/G-Star Raw CV), ECLI:EU:C:2019:721 = GRUR-Prax 2019, 464 (REDLICH). 
Cofemel - Sociedade de Vestuário SA (im Folgenden: Cofemel) und G-Star Raw CV (im Folgenden: G-Star) entwerfen, produzieren und vermarkten jeweils ua Jeans, Sweatshirts und T-Shirts. G-Star erhob im Jahr 2013 Klage gegen Cofemel wegen Verstoßes gegen Urheber- und Lauterkeitsrecht und stützte sich dabei auf eine unerlaubte Nachahmung ihres Jeansmodells «ARC» sowie des Sweat- und T-Shirt-Modells «ROWDY»:
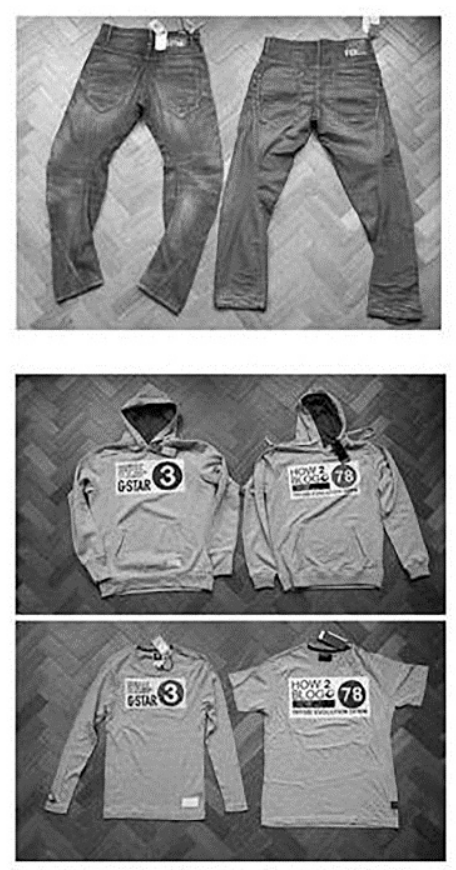

Abbildung 1. (C) www.aippi.org

Cofemel bestritt die Werkeigenschaft der geltend gemachten Erzeugnisse mangels «Schöpfungshöhe». Darüber hinaus würde das portugiesische Designrecht solchen Gebrauchsschöpfungen ohnehin einen (abgelaufenen) Musterschutz zubilligen. Ein Urheberrchtsschutz iS eines «doppelten Geistigen Eigentums» wäre damit ausgeschlossen. 
Das Erstgericht und das Berufungsgericht gaben der Klage statt. Der Oberste Portugiesische Gerichtshof hatte Zweifel und legte dem EuGH schließlich Fragen zur Auslegung vor, ob Art 2 lit a der InfoSoc-RL ${ }^{4}$ einer nationalen Vorschrift entgegensteht, nach der Modelle wie die im Ausgangsverfahren streitbefangenen Bekleidungsmodelle nur dann urheberrechtlich geschützt sind, wenn sie über ihren Gebrauchszweck hinaus einen eigenen, ästhetisch markanten visuellen Effekt hervorrufen. Mit anderen Worten: es stellte sich einmal mehr die Frage nach dem unionsrechtlichen Verständnis eines «Werkes iSd Urheberrechts».

\section{Entscheidung des Gerichts}

Die Dritte Kammer folgte dem Kumulationsprinzip von Schutzrechten, maW einer Koexistenz von nationalem Design- und europäischem Urheberrechtsschutz. ${ }^{5}$ Gewerbliche Muster und Modelle können kumulativen Schutz nach dem Gemeinschaftsgeschmacksmusterrecht und dem Urheberrecht genießen. Nach Art 96 Abs 2 Satz 1 GGV6 kann sowohl ein nicht eingetragenes als auch ein eingetragenes Unionsdesign, das nach Maßgabe der GGV geschützt ist, (auch) nach dem Urheberrecht eines Mitgliedstaates geschützt werden. ${ }^{7}$

Der EuGH lehnte eine ästhetische Originalität als grundlegendes Kriterium für die Gewährung des urheberrechtlichen Schutzes von Designs ab. Abzustellen wäre allein auf den Ausdruck der künstlerischen Freiheit. Der Begriff des «Werkes» ist ein unionsautonomer Begriff. Dieser sei einheitlich für alle Werkarten auszulegen sowie anzuwenden und habe zusammengefasst nur zwei Tatbestandsmerkmale: Zum einen

4 Richtlinie 2001/29/EG des Europäischen Parlaments und des Rates vom 22. Mai 2001 zur Harmonisierung bestimmter Aspekte des Urheberrechts und der verwandten Schutzrechte in der Informationsgesellschaft (InfoSoc-RL), ABl L 167/2001, 10.

5 Deutlich EuGH 12.9.2019, C-683/17 (Cofemel/G-Star Raw) Rz 45, ECLI:EU:C:2019:721.

6 Verordnung (EG) Nr. 6/2002 des Rates vom 12. Dezember 2001 über das Gemeinschaftsgeschmacksmuster (GGV), ABl L 3/2002, 1.

7 Einschränkend hingegen auf eingetragene Muster ausdrücklich Art 17 der Richtlinie 98/71/EG des Europäischen Parlaments und des Rates vom 13. Oktober 1998 über den rechtlichen Schutz von Mustern und Modellen (Muster-RL), ABl L 289/1998, 28; vgl. EuGH 27.1.2011, C-168/09 (Flos/Semeraro Casa e Famiglia) Rz 32 = ECLI:EU:C:2011:29. 
müsse es sich bei dem betreffenden Gegenstand um ein Original handeln, das die eigene geistige Schöpfung seines Urhebers darstellt. Zum anderen sei die Einstufung als «Werk» den Elementen vorbehalten, die eine solche Schöpfung zum Ausdruck bringen. ${ }^{8}$ Die Gestaltung müsste die freien kreativen Entscheidungen des Gestalters zum Ausdruck bringen und die Persönlichkeit des Urhebers widerspiegeln.

Die kumulative Schutztauglichkeit als urheberrechtliches Werk besteht unabhängig davon, ob das Design vor oder nach Inkrafttreten der GGV erschaffen wurde, solange die urheberrechtliche Schutzfrist noch offen ist. ${ }^{9}$ Übergangs- oder Umstellungsregelungen sind möglich, wenn sie verhältnismäßig und im Interesse des Vertrauensschutzes erfolgen. ${ }^{10}$

Wenn die Gestaltung hingegen durch technische Erwägungen, durch Regeln oder durch andere Zwänge bestimmt war, die der Ausübung künstlerischer Freiheit keinen Raum ließen, fehlte es an der erforderlichen Originalität. ${ }^{11}$

Die ästhetische Wirkung, die ein Bekleidungsmodell idR aufweist, ist dagegen naturgemäß dem subjektiven Schönheitsempfinden des jeweiligen Betrachters vorbehalten und für sich genommen als Schutzkriterium untauglich. ${ }^{12}$

8 EuGH 12.9.2019, C-683/17 (Cofemel/G-Star Raw) Rz 29, ECLI:EU:C:2019:721; näher zu den Konsequenzen daraus siehe unten Pkt. B.I.

9 EuGH 27.1.2011, C-168/09 (Flos) Rz 44, ECLI:EU:C:2011:29; vgl. auch EuGH 9.9.2011, C-198/10 (Cassina/Alivar und Galliani Host Arredamenti), ECLI:EU:C:2011:570.

10 EuGH 27.1.2011, C-168/09 (Flos/Semeraro Casa e Famiglia) Rz 55 ff und Rz 65, ECLI:EU:C:2011:29; vgl. auch EuGH 29.6.1999, C-60/98 (Butterfly Music) Rz 23 und Rz 28, ECLI:EU:C:1999:333; vgl. auch RuHL, Gemeinschaftsgeschmacksmuster Kommentar ${ }^{3}$ (2019) Art $96 \mathrm{Rz} 30$.

11 Dazu beschäftigt ein weiteres, noch unentschiedenes Vorabentscheidungsverfahren aus Belgien den EuGH in der Rs C-833/18 (Brompton Bicycle/Chedech/Get2Get); abrufbar unter https://curia.europa.eu (15.10.2019).

12 Deutlich EuGH 12.9.2019, C-683/17 (Cofemel/G-Star Raw) Rz 54, ECLI:EU:C:2019:721. 


\section{HHole/PHaradise ${ }^{13}$}

\section{Ausgangsfall}

Der Rechtsstreit um den (Fort)Bestand des sog «Mannheimer Lochs» führte gleich zu zwei höchstgerichtlichen, im Ergebnis gleichlautenden Entscheidungen zum Urheberrecht in Deutschland. ${ }^{14}$

Dabei ging es um eine künstlerische Rauminstallation im Athene Trakt der Kunsthalle Mannheim. Die Arbeit umfasste multimediale Ausgestaltungen auf allen Ebenen des Gebäudeteils, die durch vertikal angeordnete kreisförmige Öffnungen in den Geschossdecken vom Fundament bis zum Dach gedanklich miteinander verbunden sind. Von der untersten Ebene aus projizierte ein spezieller Projektor einen vertikal nach oben gerichteten Lichtstrahl, während vom Dachgeschoss Tageslicht eindrang, sodass sich das künstliche und natürliche Licht innerhalb des gleichsam virtuellen Raums trafen:

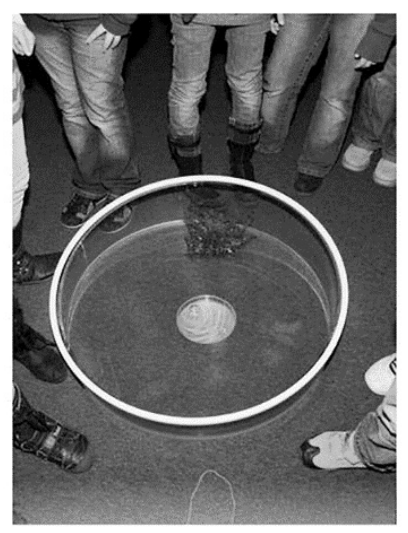

Abbildung 2. (C) Rinderspacher

BGH 21.2.2019, I ZR 98/17 (HHole [for Mannheim]), NJW 2019, 2322 = GRUR 2019, $609=$ GRUR-Prax 2019, 238 (KLAWITTER); BGH 21.2.2019, I ZR 99/17 (HHole [for Mannheim]), NJW 2019, 2331 = GRUR 2019, 621.

Dazu Bullinger/v. RAUCH, Paradigmenwechsel in der BGH-Rechtsprechung: Vollständige Vernichtung eines Werks als «andere Beeinträchtigung» im Sinne des Urheberrechts, GRUR-Prax $2019,226$. 
Für Sanierungs- und Umbaumaßnahmen wurde der Gebäudeteil, in dem die Installation angeordnet war, weitgehend entkernt und durch einen einzigen zwölf Meter hohen Raum ersetzt. Dabei wurde die Installation entfernt bzw. zerstört.

Die Künstlerin der Licht- und Rauminstallation, Nathalie Braun Barends, auch bekannt als Petsire, klagte die Kunsthalle Mannheim und verlangte ua die Wiederherstellung ihrer Arbeit, hilfsweise im Fall ihrer dauerhaften Beseitigung auch Schadenersatz. Die Vorinstanzen wiesen die Klage ab. Im Wesentlichen mit der Begründung, dass auch im Fall der vollständigen Vernichtung eines urheberrechtlich geschützen Werkes durch den (materiellen) Eigentümer eine Interessenabwägung vorzunehmen wäre. Das Interesse des Urhebers an der Fortexistenz seines Kunstwerks bei Werken der Baukunst, bei mit Bauwerken unlösbar verbundenen Kunstwerken oder sonst bei grundstücksgebundenen Kunstwerken müsse in aller Regel hinter den Interessen des Eigentümers an der anderweitigen Nutzung oder Bebauung seines Grundstücks und der damit verbundenen vollständigen Zerstörung oder Entfernung des Kunstwerks zurücktreten. ${ }^{15}$

Aufgrund der Revision der Künstlerin hatte sich der BGH mit dem seit mehr als hundert Jahren unglücklich gelösten Verhältnis zwischen geistigem und körperlichem Eigentum zu befassen. Demzufolge war der Besteller und Eigentümer eines Freskogemäldes, das sich im Treppenflur eines bewohnten Gebäudes einer Großstadt befand, nicht befugt, ohne Einwilligung des Urhebers des Gemäldes Änderungen daran vorzunehmen. ${ }^{16}$

\section{Entscheidung des Gerichts}

Die dagegen gerichtete Revision wies der I. Zivilsenat zurück, hielt aber in Abkehr von der bisherigen $\mathrm{Rsp}^{17}$ fest, dass die Vernichtung eines urheberrechtlich geschützten Werks sehrwohl eine «andere Beeinträchtigung» iSv § 14 dUrhG darstellen könne. Bei

Deutlich OLG Karlsruhe 26.4.2017, 6 U 92/15 (Permanente Rauminstallation), GRUR 2017, 803.

16 RG 8.6.1912, Rep I 382/11 (Felseneiland mit Sirenen), RGZ 79, 398 (401); vgl. auch BGH 19.3.2008, I ZR 166/05 (St. Gottfried), NJW 2008, 3784 = MDR 2008, 1351 = GRUR 2008, $984=$ RdW 2008/192, 245 = MR-Int 2008, 38; dazu THIELE, Sankt Gottfried - Rechtsvergleichende Aspekte des Architektenurheberrechts, MR-Int 2008, 36.

17 Umfassend zum frühren Meinungsstand statt vieler Dietz/PEuKERT in SCHRicker/Loewenheim (Hrsg), Urheberrecht ${ }^{4}$ (2010) § $14 \mathrm{Rz} 37$ ff mwN. 
der Prüfung, ob die Vernichtung geeignet ist, die berechtigten persönlichen und geistigen Interessen des Urhebers am Werk zu gefährden, ist eine umfassende Abwägung der Interessen des Urhebers und des Eigentümers des Werks vorzunehmen.

- Bei der Interessenabwägung ist auf Seiten des Urhebers zu berücksichtigen, ob es sich bei dem vernichteten Werk um das einzige Vervielfältigungsstück des Werks handelte, oder ob von dem Werk weitere Vervielfältigungsstücke existieren. Ferner ist zu berücksichtigen, welche Gestaltungshöhe das Werk aufweist und ob es ein Gegenstand der zweckfreien Kunst ist oder als angewandte Kunst einem Gebrauchszweck dient.

- Auf Seiten des Eigentümers können, wenn ein Bauwerk oder Kunst in oder an einem solchen betroffen ist, bautechnische Gründe oder das Interesse an einer Nutzungsänderung von Bedeutung sein. Bei Werken der Baukunst oder mit Bauwerken unlösbar verbundenen Kunstwerken werden die Interessen des Eigentümers an einer anderweitigen Nutzung oder Bebauung des Grundstücks oder Gebäudes den Interessen des Urhebers am Erhalt des Werks in der Regel vorgehen, sofern sich aus den Umständen des Einzelfalls nichts anderes ergibt.

- Im Rahmen der Interessenabwägung kann sich auswirken, ob der Eigentümer dem Urheber Gelegenheit gegeben hat, das Werk zurückzunehmen oder - wenn dies aufgrund der Beschaffenheit des Werks nicht möglich ist - Vervielfältigungsstücke hiervon anzufertigen.

Im Ergebnis verbleibt die entscheidende Bewertung vorzunehmen, ob dem Urheber eine durch die bauliche Veränderung bedingte Zerstörung seines Werks zuzumuten ist. Ob dazu weniger beeinträchtigende Lösungen in Betracht kämen, ist nicht ausschlaggebend.

\section{B. EUROPÄISCHER WERKBEGRIFF}

Die Rsp des EuGH stärkt und harmonisiert den Urheberrechtsschutz. ${ }^{18}$ Der Werkbegriff, auf den die EU-Urheberrechtsrichtlinien abstellen, erfordert zwingend eine Aus- 
drucksform des urheberrechtlichen Schutzobjekts, die es mit hinreichender Genauigkeit und Objektivität identifizierbar werden lässt, auch wenn diese Ausdrucksform nicht notwendigerweise dauerhaft sein sollte. Diese Europäische Judikatur hat große Bedeutung auch für das kreative elektronische Schaffen. ${ }^{19}$

\section{Urheberrechtliches Werk iS des Unionsrechts}

Für die Einstufung eines Objekts als «Werk» iSd InfoSoc-RL müssen insoweit zwei kumulative Voraussetzungen erfüllt sein. Zum einen muss das betreffende Objekt eine eigene geistige Schöpfung seines Urhebers darstellen. Zum anderen müssen Elemente vorliegen, die eine solche geistige Schöpfung zum Ausdruck bringen. ${ }^{20}$

\section{Merkmal der objektiven Identifizierung}

Das Urheberrecht kann nur für ein jeweiliges Schutzobjekt angewendet werden, bei dem es sich um ein Original in dem Sinne handelt, dass es eine eigene geistige Schöpfung seines Urhebers darstellt. ${ }^{21}$ Nach ErwGr 15 der Software-RL sind nach dem Recht und der Rechtsprechung der Mitgliedstaaten sowie nach den internationalen Urheberrechtskonventionen die Ausdrucksform von Ideen und Grundsätze urheberrechtlich zu schützen. ${ }^{22}$ Im internationalen Recht sehen sowohl Art 2 WCT als auch Art 9 Abs 2 TRIPS-Abk vor, dass sich der urheberrechtliche Schutz auf Ausdrucksformen, nicht aber auf Ideen, Verfahren, Arbeitsweisen oder mathematische Konzepte als solche erstreckt. $^{23}$

An der Möglichkeit einer präzisen und objektiven Identifizierung fehlt es aber im Fall des Geschmacks eines Lebensmittels. Im Unterschied etwa zu einem literarischen, bild-

19 Vgl. dazu bereits THIELE, Das urheberrechtliche Werk ist keine Frage des Geschmacks, jusIT 2018/77, $214 \mathrm{mwN}$.

20 EuGH 16.7.2009, C-5/08 (Infopaq I) Rz 37 und 39, ECLI:EU:C:2009:465.

21 EuGH 16.7.2009, C-5/08 (Infopaq I) Rz 35, ECLI:EU:C:2009:465; EuGH 1.12.2011, C-145/10 (PAINER) Rz 87, ECLI:EU:C:2013:138.

22 Vgl EuGH 2.5.2012, C-406/10 (SAS-Institute) Rz 32, ECLI:EU:C:2012:259.

23 EuGH 2.5.2012, C-406/10 (SAS-Institute) Rz 33, ECLI:EU:C:2012:259. 
nerischen, filmischen oder musikalischen Werk beruht die Identifizierung des Geschmacks eines Lebensmittels nämlich im Wesentlichen auf Geschmacksempfindungen und -erfahrungen, die subjektiv und veränderlich sind, da sie ua von Faktoren abhängen, die mit der Person verbunden sind, die das betreffende Erzeugnis kostet, wie etwa deren Alter, Ernährungsvorlieben und Konsumgewohnheiten, sowie von der Umwelt oder dem Kontext, in dem das Erzeugnis gekostet wird. ${ }^{24}$

Da mit technischen Mitteln beim gegenwärtigen Stand der Wissenschaft eine genaue und objektive Identifizierung des Geschmacks eines Lebensmittels nicht möglich ist, die es erlaubte, ihn vom Geschmack anderer gleichartiger Erzeugnisse zu unterscheiden, verneint die Große Kammer eine Werkeigenschaft. Diese Gedankenführung ist zwanglos auch auf die Beurteilung technischer Sachverhalte beim Werkschaffen zu übertragen. Sie bedeutet, dass für elektronische Erzeugnisse stets eine genaue und objektive Identifizierung - letztlich durch den Source Code - vorhanden ist, sodass diese Voraussetzung des europäischen Werkbegriffes idR erfüllt wird.

\section{Kriterium der Originalität}

Aus ErwGr 16 der Datenbank-RL geht hervor, dass der Begriff der eigenen geistigen Schöpfung des Urhebers auf das Kriterium der Originalität verweist. ${ }^{25}$ Die Originalität 
ist auch das einzige Kriterium bei der Beurteilung, ob eine Datenbank für den urheberrechtlichen Schutz nach dieser Richtlinie in Betracht kommt. ${ }^{26}$

Aus ErwGr 17 der Schutzdauer-RL ergibt sich, dass es sich immer dann um eine eigene geistige Schöpfung des Urhebers handelt, wenn darin seine Persönlichkeit zum Ausdruck kommt. ${ }^{27}$ Dies ist dann der Fall, wenn der Urheber bei der Herstellung des Werks seine schöpferischen Fähigkeiten zum Ausdruck bringen konnte, indem er frei kreative Entscheidungen trifft. ${ }^{28}$

So kann ein Objekt (zB Fotografie) urheberrechtlich geschützt sein, sofern es, was das nationale Gericht im Einzelfall zu prüfen hat,

- die eigene geistige Schöpfung des Urhebers darstellt,

- in der dessen Persönlichkeit zum Ausdruck kommt und

- die sich in dessen bei ihrer Herstellung getroffenen freien kreativen Entscheidungen ausdrückt. ${ }^{29}$

Dabei ist der Schutz eines Objekts (zB Porträtfotografie) nicht schwächer als derjenige, der anderen Werken, insb anderen fotografischen Werken, zukommt. ${ }^{30}$

Für die Erstellung einer Datenbank ist das Kriterium der Originalität erfüllt, wenn ihr Urheber über die Auswahl oder Anordnung der in ihr enthaltenen Daten seine schöpferischen Fähigkeiten in eigenständiger Weise zum Ausdruck bringt, indem er freie und kreative Entscheidungen trifft, 31 und ihr damit seine "persönliche Note» verleiht. ${ }^{32}$ Dagegen ist dieses Kriterium nicht erfüllt, wenn die Erstellung der Datenbank

EuGH 1.3.2012, C-604/10 (Football Dataco) Rz 40, ECLI:EU:C:2012:115; EuGH 12.9.2019, C-683/17 (Cofemel/G-Star Raw) Rz 39, ECLI:EU:C:2019:721.

EuGH 1.12.2011, C-145/10 (Painer) Rz 88, ECLI:EU:C:2011:631.

EuGH 1.12.2011, C-145/10 (Painer) Rz 89, ECLI:EU:C:2013:138; vgl im Umkehrschluss EuGH 4.10.2011, C-403/08 und C-429/08 (FAPL/Murphy) Rz 98, ECLI:EU:C:2011:631.

Vgl. EuGH 12.9.2019, C-683/17 (Cofemel/G-Star Raw) Rz 35, ECLI:EU:C:2019:721 mwN.

EuGH 1.12.2011, C-145/10 (Painer) Rz 98, ECLI:EU:C:2011:631.

Vgl EuGH 16.7.2009, C-5/08 (Infopaq I) Rz 45, ECLI:EU:C:2009:465; EuGH 22.12.2010, C-393/09 (Bezpecnostní softwarová asociace) Rz 50, ECLI:EU:C:2010:816; EuGH 1.12.2011, C-145/10 (Painer) Rz 89, ECLI:EU:C:2011:631.

EuGH 1.3.2012, C-604/10 (Football Dataco) Rz 38, ECLI:EU:C:2012:115; EuGH 1.12.2011, C-145/10 (Painer) Rz 92, ECLI:EU:C:2011:631. 
durch technische Erwägungen, Regeln oder Zwänge bestimmt wird, die für künstlerische Freiheit keinen Raum lassen. ${ }^{33}$ Demzufolge steht Art 3 Abs 1 Datenbank-RL nationalen Rechtsvorschriften entgegen, durch die Datenbanken, die unter die Definition des Art 1 Abs 2 Datenbank-RL fallen, unter anderen Voraussetzungen als der in Art 3 Abs 1 leg cit aufgestellten Voraussetzung der Originalität urheberrechtlicher Schutz gewährt wird. ${ }^{34}$

\section{Abgrenzungen zu Nicht-Werken}

Der Werkbegriff des Europäischen Urheberrechts gewinnt mit der eingangs aufgegriffenen Entscheidung weiter an Kontur. In einer Reihe von Urteilen hat sich demnach der EuGH ausdrücklich dazu geäußert, was kein Werk iSd InfoSocRL darstellen kann:

- So können Sportereignisse als solche nicht als geistige Schöpfungen angesehen werden, die sich als Werke einordnen ließen. Das gilt insb für Fußballspiele, die Spielregeln unterliegen, die für eine künstlerische Freiheit iSd Urheberrechts keinen Raum lassen. Daher können Sportereignisse keinen urheberrechtlichen Schutz genießen. ${ }^{35}$

Im Levola-Urteil ${ }^{36}$ bestimmte die Große Kammer deutlich die Anforderungen an die Körperlichkeit des Werkbegriffes. Zum einen müssen nämlich die Behörden, die mit dem Urheberrechtsschutz betraut sind, die geschützten Objekte klar und genau erkennen können. Dasselbe gilt für Privatpersonen, insb Wirtschaftsteilnehmer, die mit Klarheit und Genauigkeit die Objekte identifizieren können müssen, die zugunsten von Dritten, insb Wettbewerbern, geschützt sind. Zum anderen impliziert das Erfordernis des Ausschlusses jedes - der Rechtssicherheit schädlichen - subjektiven Elements bei der Identifizierung des geschützten Objekts, dass dieses Gegenstand eines präzisen und objektiven Ausdrucks sein kann. ${ }^{37}$ 22.12.2010, C-393/09 (Bezpecnostní softwarová asociace) Rz 48 und 49, ECLI:EU:C:2010:816; EuGH 4.10.2011, C-403/08 und C-429/08 (FAPL/Murphy) Rz 98, ECLI:EU:C:2011:631. 
- Nach der Entscheidung der Luxemburger Richter ${ }^{38}$ haben auch Parfümhersteller kaum Chancen, ihre Gerüche urheberrechtlich durchzusetzen.

- Erzeugnisse wie etwa Kleidungsstücke, die selbst über ihren bloßen Gebrauchszweck hinaus einen eigenen, ästhetisch markanten visuellen Effekt hervorrufen, sind nicht als «Werke» iSd InfoSoc-RL einzustufen. 39

Darzulegen und im Streitfall unter Beweis zu stellen ist daher, ob ein bestehender künstlerischer Gestaltungsspielraum besteht und genutzt wird, der den schöpferischen Geist des Urhebers in origineller Weise zum Ausdruck zu bringen vermag. ${ }^{40}$ Die ästhetische Wirkung von gewerblichen Mustern und Modellen bleibt im Rahmen der Prüfung der Urheberrechtsfähigkeit dagegen weiter unberücksichtigt. Wird ein vorhandenes Vorbild nur geringfügig ohne künstlerische Leistung geändert, scheidet der Urheberechtschutz weiterhin aus.

Als Zwischenergebnis lässt sich daher festhalten: Die Kreativen in der Softwarebranche sowie im Webdesign gehen urheberrechtlich gestärkt aus den bisher vorliegenden Entscheidungen zur Negativabgrenzung europäischer Werke hervor.

\section{MITGLIEDSTAATLICHER WERKSCHUTZ}

Das Recht auf Werkintegrität nach § 21 UrhG stellt ein Persönlichkeitsrecht des Urhebers dar. Es schützt sein Werk iwS vor unbefugten Veränderungen in der Öffentlichkeit. Originale der bildenden Kunst schützt § 21 Abs 2 UrhG sogar im privaten Bereich. $\S 21$ Abs 3 UrhG sieht bei Entstellungen, Verstümmelungen und anderen grob verletzenden Werkänderungen ein absolutes Änderungsverbot vor.

EuGH 13.11.2018, C-310/17 (Levola Hengelo/Smilde Foods), ECLI:EU:C:2018:899.

EuGH 12.9.2019, C-683/17 (Cofemel/G-Star Raw) Rz 55, ECLI:EU:C:2019:721.

Der Urheberrechtsschutz für Modebekleidung reduziert sich praktisch auf einzelne, aufwändig gestaltete Einzelstücke, scheidet aber für Massentextilien von vornherein aus. 


\section{Unionsrechtliche Grundlagen}

Der Unionsgesetzgeber erwähnt zwar das Urheberpersönlichkeitsrecht im Richtlinienrecht, so wie etwa ausdrücklich in Art 9 Schutzdauer-RL ${ }^{41}$ samt ihrem ErwGr 21, im ErwGr 28 der Satelliten- und Kabel-RL, ${ }^{42}$ im ErwGr 28 der Datenbank-RL ${ }^{43}$ sowie ErwGr 19 der InfoSoc-RL, hat es in seine Harmonisierungstätigkeit aber bislang nicht einbezogen. Das WCT ${ }^{44}$ bzw. WPPT ${ }^{45}$ gelten aber als Sonderabkommen iSv Art 20 RBÜ46 und dürfen daher keinen geringeren Schutz als Art 6bis RBÜ bieten, der den Entstellungsschutz verbrieft. Die gesetzgeberische Entscheidung der EU steht im Einklang mit der Berner Übereinkunft, an deren Art 1 bis 21 sich die Union, obwohl sie nicht Vertragspartei der Berner Übereinkunft ist, nach Art 1 Abs 4 WCT, dem sie beigetreten ist, halten muss. ${ }^{47}$ Konkret verweist Art 1 Abs 4 WCT auf bzw. benennt Art 5 WPPT das Urheberpersönlichkeitsrecht. Nach der Ratifizierung beider Verträge gehört das Urheberpersönlichkeitsrecht zum acquis communautaire. Gleichwohl handelt es sich beim Entstellungsschutz nicht um einen harmonisierten Bereich, sodass Wertungen des nationalen Grundrechtsschutzes durchaus Berücksichtigung finden können. ${ }^{48}$

41 Richtlinie 93/98/EWG des Rates vom 29. Oktober 1993 zur Harmonisierung der Schutzdauer des Urheberrechts und bestimmter verwandter Schutzrechte (Schutzdauer-RL), ABl L 290/1993, 9.

42 Richtlinie 93/83/EWG des Rates vom 27. September 1993 zur Koordinierung bestimmter urheber- und leistungsschutzrechtlicher Vorschriften betreffend Satellitenrundfunk und Kabelweiterverbreitung (Satelliten- und Kabel-RL), ABl L 248/1993, 15.

43 Richtlinie 96/9/EG des Europäischen Parlaments und des Rates vom 11. März 1996 über den rechtlichen Schutz von Datenbanken (Datenbank-RL), ABl L 77/1996, 20.

44 WIPO Copyright Treaty (WCT) - WIPO Vertrag über Darbietungen und Tonträger, ABl L 2000/98, 8.

45 WIPO Performances and Phonograms Treaty (WPPT) - Wipo Vertrag über Darbietungen und Tonträger, ABl L 2000/98, 15

46 (Revidierte) Berner Übereinkunft zum Schutzvon Werken der Literatur und Kunst samt Anhang (Pariser Fassung), BGBl 1982/319 idF 1985/133 und BGBl 1986/612.

Vgl. EuGH 12.9.2019, C-683/17 (Cofemel/G-Star Raw) Rz 41, ECLI:EU:C:2019:721; EuGH 13. 11. 2018, C-310/17 (Levola Hengelo/Smilde Foods) Rz 38, ECLI:EU:C:2018:899. wbl 2019/149, 476 = ZIIR Slg 2019/45: zur Rechtfertigung durch die Meinungsfreiheit nach Art 10 EMRK. 
Die Europäische Rsp hat darüber hinaus bereits anerkannt, dass dem Urheber grundsätzlich ein berechtigtes Interesse daran zukommt, dass sein geschütztes Werk nicht mit diskriminierenden Aussagen bzw. Herabwürdigungen entgegen Art 21 Abs 1 GRC in Verbindung gebracht wird. 49

Als Zwischenergebnis ist daher festzuhalten, dass die Regelung des Entstellungsschutzes eines urheberrechtlich geschützten Werkes bis hin zu seiner völligen Zerstörung durch den Eigentümer - nach derzeitigem Stand der Harmonisierung im Unionsurheberrecht - den mitgliedstaatlichen Rechtsordnungen vorbehalten bleibt.

\section{Verletzung von $§ 21$ UrhG - Kein Zerstörungsschutz in Österreich?}

Begibt sich der Urheber seines geschützten Werkes durch Veräußerung an den Kunstmarkt, überträgt er damit letztlich Besitz und Eigentum an einen Dritten. Dieser materielle Eigentümer kann nach $§ 362$ ABGB «kraft des Rechtes, frey über sein Eigenthum zu verfügen» die Sache «nach Willkühr benützen oder unbenützt lassen; er kann sie vertilgen, ganz oder zum Theile auf Andere übertragen, oder unbedingt sich derselben begeben, das ist, sie verlassen». Im Ergebnis darf nach der österreichischen Gesetzeslage und bisherigen Rechtspraxis der Eigentümer ein erworbenes Ölbild zwar nicht einmal in seiner Privatspähre nach § 21 Abs 2 UrhG verändern, wohl aber vollständig vernichten. 50

\section{Grundlagen und Regelungsgehalt}

$\S 21$ UrhG lautet nach wie vor:51

«(1) Wird ein Werk auf eine Art, die es der Öffentlichkeit zugänglich macht, benutzt oder zum Zweck der Verbreitung vervielfältigt, so dürfen auch von dem zu

49 EuGH 3.9.2014, C-201/13 (Deckmyn und Vrijheidsfonds] Rz 30 f, ECLI:EU:C:2014:2132 = ÖJZ 2014/133, 890 (LEHOFER) = EuGRZ 2014, 536 = jusIT 2014/78, 165 (THIELE) = wbl 2014/234, 701 = ecolex 2014/416, 982 (SCHUMACHER) = ecolex 2014, $1025=$ MR-Int 2014, $124=$ ÖBl 2014/58, 282 (HANDIG); dazu THIELE, De Wilde Weldoener - Auswirkungen der Europäischen Parodiefreiheit auf das deutsche und österreichische Urheberrecht, in: SCHWEIGHOFER/ KuMMER/HöTZENDORFER (Hrsg), Kooperation (2015), 639.

50 Deutlich Toms in Kucsko/HANDIG (Hrsg), urheber.recht² (2017) § $21 \mathrm{Rz} 30$.

51 Stammfassung BGBl 111/1936. 
einer solchen Werknutzung Berechtigten an dem Werke selbst, an dessen Titel oder an der Urheberbezeichnung keine Kürzungen, Zusätze oder andere Änderungen vorgenommen werden, soweit nicht der Urheber einwilligt oder das Gesetz die Änderung zuläßt. Zulässig sind insbesondere Änderungen, die der Urheber dem zur Benutzung des Werkes Berechtigten nach den im redlichen Verkehr geltenden Gewohnheiten und Gebräuchen nicht untersagen kann, namentlich Änderungen, die durch die Art oder den Zweck der erlaubten Werknutzung gefordert werden.

(2) Für Urstücke von Werken der bildenden Künste gelten die Vorschriften des Absatzes 1 auch dann, wenn die Urstücke nicht auf eine Art benutzt werden, die das Werk der Öffentlichkeit zugänglich macht.

(3) Die Erteilung der Einwilligung zu nicht näher bezeichneten Änderungen hindert den Urheber nicht, sich Entstellungen, Verstümmelungen und anderen Änderungen des Werkes zu widersetzen, die seine geistigen Interessen am Werke schwer beeinträchtigen.»

Die Vorschrift soll durch ein grundsätzliches Änderungsverbot gewährleisten, dass Teil-Werknutzungen, Änderungen, Umgestaltungen, Beeinträchtigungen oder Entstellungen des Werkes der Zustimmung des Urhebers bedürfen. ${ }^{52}$

$\S 21$ bezweckt nicht nur den allgemeine Persönlichkeitsschutz von Ruf und Ehre, die in Art. 6bis Abs 1 RBÜ genannt werden, sondern den umfassenderen Schutz des geistigen und persönlichen Bandes, das zwischen dem Urheber und seinem Werk besteht. 53

Von seinem Regelungsgehalt her schützt § 21 Abs 1 UrhG die Integrität in sämtlichen Werkkategorien bei jeder Handlung, die das Werk der Öffentlichkeit zugänglich macht. Demgegenüber erfasst \ 21 Abs 2 UrhG die Werkintegrität für die bildende Kunst auch ohne Öffentlichkeitserfordernis. § 21 Abs 3 UrhG enthält ein absolutes Änderungsverbot. Gegen Entstellungen und Beeinträchtigungen ihrer Werke sind auch sämtliche

OGH 22.6.1999, 4 Ob 159/99g (Zimmermann FITNESS), MR 1999, 282 = ÖBl 2000, 130 (KUCSKO). wbl 2019/149, 476 = ZIIR-Slg 2019/45. 
ausländischen Staatsangehörigen geschützt, selbst wenn sie nicht Angehörige von Mitgliedstaaten der einschlägigen internationalen Konventionen sind, aber die fremdenrechtlichen Anknüpfungspunkte der Gleichrangigkeit oder Staatsverträge erfüllt sind. ${ }^{54}$

Der Werkschutz bei gewerbsmäßig hergestellten Filmwerken findet sich in $\S \S 38$ Abs 2, 39 Abs 3 und wird im Folgenden nur insoweit einbezogen, als er für das Verständnis des Schutzes eines urheberrechtlichen Werkes vor seiner Zerstörung durch den Eigentümer von Relevanz ist. Schließlich ist bei der Inanspruchnahme von freien Werknutzungen ist $§ 57$ Abs 1 UrhG zu beachten, der eine Urhebernennung vorsieht und unionsrechtlich hinterlegt ist. 55

\section{Begriff des Werkschutzes}

Die vom österreichischen Gesetzgeber gewählte Überschrift «Werkschutz» scheint einen objektiven Schutz des Werkes, ähnlich dem Denkmalschutz, anzukündigen. Tatsächlich aber geht es um das Recht des Urhebers zu bestimmen, in welcher Form sein Werk der Öffentlichkeit entgegentritt, 56 maW um den Urheberpersönlichkeitsschutz.

Wenn das Werk den Stempel der Persönlichkeit seines Urhebers trägt, so wirkt jede Änderung des Werkes als Beeinträchtigung der Schöpferpersönlichkeit und bedarf daher der Einwilligung des Urhebers. ${ }^{57}$

\section{Schutz der Werkintegrität auch ohne Öffentlichkeit}

In § 21 Abs 2 UrhG regelt der Gesetzgeber den Fall, dass ein Urstück eines Werks der bildenden Kunst auf urheberrechtlich relevante Art ohne Zustimmung des Urhebers

54 Vgl. § 96 UrhG.

55 Vgl. EuGH 1.12.2011, C-145/10 (Painer), ECLI:EU:C:2013:138.

56 OGH 8.3.1994, 4 Ob 165/93 (Das österreichische Recht), EvBl 1994/103 = MR 1994, 117 (WALTER) $=$ ÖBl 1994, $182=$ ARD 4582/29/94: Urheberpersönlichkeitsrecht.

57 Vgl deutlich für Lichtbildwerke: OGH 12.9.2001, 4 Ob 179/01d (Eurobike), MR 2001, 389 (WALTER) $=$ RdW 2002/205, 217 = MR 2002, 195 (SWOBODA) = ÖBl 2003/12, 39 (GAMERITH); dazu NoLL, Reduzierter Europäischer Werkbegriff für Lichtbildwerke («Eurobike»), ÖBl 2003, 164. 
verändert wird. Hier geht er von dem sonstigen Erfordernis der Öffentlichkeit ab. Er begründet es mit der Gefahr, dass das so geänderte Urstück eines Werks der bildenden Kunst wegen der Unmöglichkeit, sein künftiges Schicksal vorauszusehen, in dieser geänderten Form der Öffentlichkeit zugänglich wird.58

Nach dem Wortlaut des § 21 Abs 2 UrhG sind daher das verkürzte Abschreiben eines Gedichtes und das Klavierspiel einer geänderten Version in den eigenen vier Wänden immer zulässig, da andere Werkkategorien vorliegen.

Auf die Sonderregelung des § 83 UrhG, insbesondere zum Ausschilderungsanspruch, die sich mit den Folgen der Änderung eines Urstücks eines Werks der bildenden Kunst befasst, wird an dieser Stelle aus Platzgründen lediglich verwiesen. ${ }^{59}$

\section{Zulässige Einschränkungen}

Das österreichische Gesetz gestattet aber branchenübliche «Änderungen» in den Fällen des $§ 21$ Abs 1 und Abs 2 UrhG.

\subsection{Branchenübliche Änderungen}

§ 21 Abs 1 Satz 2 UrhG wählt dafür die Formulierung der Auslegungsregel im § 914 ABGB «Übung des redlichen Verkehrs». Anerkannt dabei ist, dass die Korrektur von Schreibfehlern und Interpunktionen im Romanmanuskript eines Autors durch den Verleger zulässig sind (wohl auch Änderungen der Rechtschreibung nach neuen Regeln) oder die Kürzung von Leserbriefen durch den Herausgeber einer Zeitung. ${ }^{60}$ Ob dieser - vom Gesetzgeber so gewollte - unbestimmte Gesetzeswortlaut die radikale Neuinszenierung eines Schauspiels einschließt, samt dazu erforderlicher

OGH 11.5.2010, 4 Ob 49/10z (Tirol Milch-Logo), ecolex 2010/287, 781 (SCHÖNHERR/GRÖTSCHL) = ÖBl-LS 2010/165/166 = MR 2010, $268=$ RdW 2010/653, 632 = HS 41.185. von ihm geschaffenen Bauwerke. Die Abwägung der Urheber- und Eigentümerinteressen bei Umbau von oder Zubau zu Baukunstwerken, ipCompetence 2014 H 11, 56 mwN. 
Textänderungen, ist eine offene Frage, die nur von Fall zu Fall beantwortet werden kann. ${ }^{61}$

Das Gebot der Werktreue sollte also generell, wenn schon nicht in künstlerischer, so doch aus urheberrechtlicher Sicht, Vorrang haben. ${ }^{62}$

In folgenden Fällen rechtfertigt die Übung des redlichen Verkehrs einen Eingriff in die Werkintegrität:

- So werden zum Zweck der besseren Verwertung und Vermarktung vorhandene Filme durch Verlängerung oder Kürzung den im Fernsehen zur Verfügung stehenden Sendezeiten bzw der programmunterbrechenden Werbung angepasst.63 Das kann durch schnelleres oder langsameres Abspielen oder durch Kürzung des Films geschehen. Auch Formatänderungen, etwa für die Wiedergabe eines Films auf dem Bildschirm eines Flugzeugs («Airline Editing») werden vorgenommen.

- Wenn der Eigentümer einer Sammlung von Kunstwerken (iSv § 6 UrhG) diese vergrößert oder verkleinert. ${ }^{64}$

- Bei Sammelwerken kommt dem ehemaligen Herausgeber (Urheber iSv § 6 UrhG) auch nach seinem Ausscheiden so lange das Nennungsrecht zu, als diese Auflagen von seinem Wirken als Herausgeber geprägt sind. 65

\subsection{Grenzüberschreitungen}

Als eine nicht mehr zulässige Einschränkung wird angesehen

- die Kolorierung eines Schwarzweißfilms, nämlich des Klassikers «Asphalt Jungle» von John Houston, 66

61 Vgl. LG Salzburg 10.3.2003, 53 R 417/02h (Fledermaus), JBl 2003, 587 = MR 2003, 391 (WALTER); dazu HollaEnder, Betrug durch «Die Fledermaus»? Das Strafrecht im Spannungsverhältnis zur Freiheit der Kunst, ÖJZ 2004/50, 797.

62 So unterstützenswert Dillenz/GUTmAnN, UrhG \& VerwGesG² (2004) § 21 Rz 5; vgl. auch WALTER, Österreichisches Urheberrecht Handbuch I (2008) Rz 947.

63 Vgl. ThiELE, Werbepausen in Fernsehfilmen: Ein Musterprozess in Schweden, MR-Int 2006, 141.

64 OGH 11.2.1997, 4 Ob 17/97x (Wiener Aktionismus), MR 1997, 98 (WALTER) = ecolex 1997, 419 $=$ ÖBl 1997, 301 .

65 OGH 8.3.1994, 4 Ob 165/93 (Das österreichische Recht), EvBl 1994/103 = MR 1994, 117 $($ WALTER $)=$ ÖBl 1994, $182=$ ARD 4582/29/94.

66 REUPERT, Der Film im Urheberrecht (1995), 141. 
- das Herausschneiden von Szenen aus Filmwerken, wenn es wegen Demonstrationen im Publikum geschieht. ${ }^{67}$

\subsection{Digitale Werkveränderungen}

Die Digitalisierung macht es aber auch möglich, dass Personen, über die genug Filmmaterial besteht, sich plötzlich in einem neuen Film vorfinden, der vorhandene Filme kombiniert (King Kong tritt in einem Charly Chaplin Film auf) oder mit dem digitalen Material über Personen völlig neue Handlungen konstruiert, sodass etwa eine Lovestory zwischen Adolf Hitler und Marilyn Monroe entsteht. Schauspieler fragen sich, ob ihr erster Auftritt in einem Film auch bereits ihr letzter war, da Aussehen, Stimme und Bewegung eingescannt, digitalisiert und in beliebiger Form für künftige Filme verwendet werden können. Tatsache ist, dass durch die Digitalisierung von Werken eine besondere Verletzlichkeit gegen Veränderung des Werks begründet wird. Diese Änderungsmöglichkeiten beinhalten, neben allen anderen berechtigten Fragen der Verwertungsrechte im digitalen Umfeld, einen persönlichkeitsrechtlichen Aspekt, der nicht zu vernachlässigen ist. ${ }^{6}$

Wie ein Teil der Lehre ${ }^{69}$ aber zutreffend am Beispiel der Skulptur «Fearless Girl» Aug in Aug mit dem «Charging Bull» vor der New Yorker Börse in der Wall Street aufzeigt, muss die Latte der unzulässigen Beeinträchtigung in einem solchen Fall durchaus hoch liegen, weil die Freiheit eines Künstlers nur dann gewahrt ist, wenn Gleiches auch für seinen Nachbarn gilt. Als Kriterien der gebotenen Interessenabwägung in diesem Zusammenhang sind vor allem die Art und die Intensität des Eingriffs, der Grad der schöpferischen Eigenart des Werks und Verwertungszweck und Verwertungsgebiet zu berücksichtigen. ${ }^{70}$ 


\section{Absolutes Änderungsverbot - Entstellungsschutz}

Es ist üblich, dass durch Formularverträge der Urheber seine Zustimmung zu später erfolgenden Änderungen eines Werkes vorweg einräumt. \$ 21 Abs 3 UrhG versucht hier ein Sicherheitsnetz einzuziehen. Dieses würde dann wirken, wenn etwa der Melodie eines geistlichen Liedes durch den Verleger ein pornographischer Text unterlegt wird oder ein Antikriegsstück durch Abänderung der Grundtendenz in sein Gegenteil verkehrt und zum Völkerhass aufrufen würde. ${ }^{71}$

Eine andere absolute Grenze kann man aus dem zweiten Satz des $§ 57$ Abs 1 UrhG ableiten: «Sinn und Wesen des benutzten Werkes dürfen in keinem Fall entstellt werden.» Diese an sich nur im Zusammenhang mit dem Schutz geistiger Interessen bei freien Werknutzungen enthaltene Bestimmung lässt sich logisch nur für alle Werknutzungen insgesamt anwenden. ${ }^{72}$ Es gäbe keinen Grund, diese absolute Grenze nur für den Fall der zustimmungsfreien Nutzung des Werkes, nicht aber auch für den Fall der zustimmungspflichtigen Nutzung, wo also die Rechte des Urhebers nicht eingeschränkt sind, anzuwenden.

Eine Entstellung des Werkes liegt also beispielswese in folgenden Fällen vor:

- Wird ein Fernsehfilm etwa um ein Viertel gekürzt und greift diese Kürzung massiv in die geistigen Interessen des Filmurhebers ein, ist sogar immaterieller Schadenersatz zu leisten. ${ }^{73}$

- Eine Beeinträchtigung der geistigen Interessen eines Autors durch massive Bearbeitung eines von ihm verfassten TV-Drehbuches durch die Sendeanstalt.74

71 Vgl. auch EuGH 3.9.2014, C-201/13 (Deckmyn und Vrijheidsfonds] Rz 30 und 31, ECLI:EU:C:2014:2132.

72 OGH 25.4.2019, 4 Ob 250/18w (Draußen bleiben), MR 2019, 126 (WALTER) = ZTR 2019, $103=$ wbl 2019/149, 476 = ZIIR-Slg 2019/45.

73 OGH 10.11.1998, 4 Ob 281/98x (Den Kopf zwischen den Schultern), MR 1998, 345 (WALTER) = JUS Z/2665.

74 OGH 3.10.1972, 4 Ob 343/72 (C'est la vie), ÖBl 1973, 112 = SZ 45/102. 


\section{Schutz vor der Zerstörung des Werkes}

\subsection{Werkvernichtung}

Die radikalste Änderung eines Werkes ist seine Zerstörung. Das österreichische Urheberrechtsgesetz, das auch beim Schutz der Werkintegrität versucht, die Interessen des körperlichen Eigentümers mit den Interessen des geistigen Eigentümers in Einklang zu bringen, verbietet dem körperlichen Eigentümer nicht, das Werk zu vernichten. Das ist, insbesondere bei Originalwerken der bildenden Kunst, sicher ein Wertungswiderspruch. Ein Ölbild etwa darf von seinem Eigentümer nach § 21 Abs 2 UrhG nicht einmal in seiner Privatsphäre verändert werden, wohl aber vernichtet werden. ${ }^{75}$

Die Zerstörung eines Kunstwerkes durch den Eigentümer sollte nach der hier vertretenen Auffassung unter § 21 UrhG fallen und unter Umständen Schadenersatz nach sich ziehen. Letzteres jedoch nur dann, wenn eine Interessenabwägung zugunsten des Urhebers ausfällt.

\subsection{Rechtslage in Deutschland}

Die dt Rsp subsumiert nunmehr - wie im Ausgangsfall zum Mannheimer Loch dargeselegt - die Zerstörung eines durch das Urheberrecht geschützten Kunstwerkes zwar nicht unter den Begriff der «Entstellung», jedoch stelle sie «eine andere Beeinträchtigung» iSv § 14 dUrhG dar. ${ }^{76}$ Durch eine Abwägung, die entweder die Interessen des Eigentümers oder die des Urhebers als gewichtiger ansehen wird, habe das Kammergericht den Rest des Gesetzestextes zu beurteilen. Nämlich, ob die Beeinträchtigung auch geeignet ist, die berechtigten geistigen oder persönlichen Interessen der Künstler am Werk zu gefährden. Sofern die Interessenabwägung zugunsten der Kläger ausgehen sollte, wird das Kammergericht weiter zu prüfen haben, ob es sich um eine schwerwiegende Verletzung des Urheberpersönlichkeitsrechts handelt, die nicht durch andere Weise als durch eine Geldentschädigung ausgeglichen werden kann.

76 Vgl. auch BGH 21.2.2019, I ZR 15/18 (Minigolfanlage), NJW 2019, $2331=$ GRUR 2019, 619. 


\subsection{Schweizer Rechtslage}

Einen anderen Weg ist hier das schweizerische Urheberrechtsgesetz gemäß Art 15 SchwURG gegangen, das zwar ebenfalls kein Verbot der Vernichtung von Originalwerken vorsieht, jedoch die Verpflichtung des Eigentümers eines Werkes vorsieht, dieses vor der beabsichtigten Zerstörung dem Urheber zur Rücknahme anzubieten. ${ }^{77}$

\section{ZUSAMMENFASSUNG}

Zusammenfassend hält die Europäische Rsp zum unionsrechtlichen Werkbegriff fest, dass dafür zwei Voraussetzungen erfüllt sein müssen, nämlich das Merkmal der objektiven Identifizierung sowie das Kriterium der Originalität. Auf eine bloß ästhetische Wirkung kommt es nicht an. Daraus folgt für den Bereich des kreativen elektronischen Schaffens ein weitgehender Schutz durch das Europäische Urheberrecht. Die nach wie vor in der Kompetenz der Mitgliedstaaten liegende urheberpersönlichkeitsrechtliche Komponenete des Werkschaffens darf nicht dazu führen, die Werkintegrität soweit zu untergraben, dass eine beliebige Zerstörung des Werkes durch den materiellen Eigentümer zu dulden ist. Die jünsgte Rsp in den Mitgliedstaaten ist um einen interessengerechten Ausgleich bemüht, der durchaus in die Auslegung der österreichischen Vorschrift zum Werkschutz nach § 21 UrhG Eingang finden sollte.

77 Statt vieler MÜLLER/ÖRTLI (Hrsg), Urheberrechtsgesetz (URG) - Kommentar² (2012), 130 ff. 
